\title{
Achieving Universal Health Coverage by Focusing on Primary Care in Japan: Lessons for Low- and Middle- Income Countries
}

\section{Naoki Ikegami*}

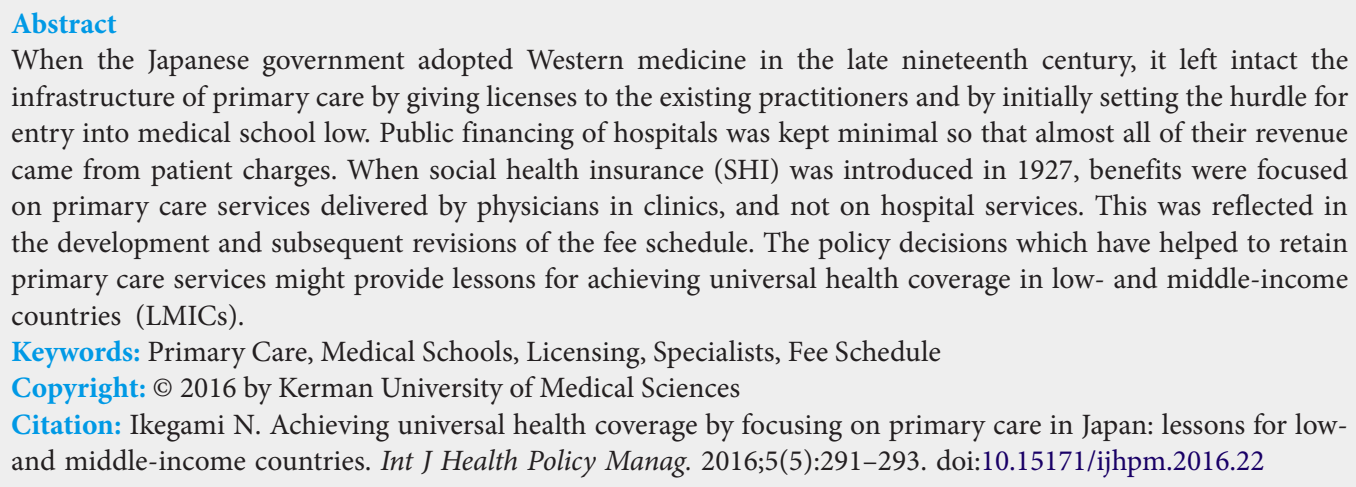

When the Japanese government adopted Western medicine in the late nineteenth century, it left intact the infrastructure of primary care by giving licenses to the existing practitioners and by initially setting the hurdle for entry into medical school low. Public financing of hospitals was kept minimal so that almost all of their revenue came from patient charges. When social health insurance (SHI) was introduced in 1927, benefits were focused on primary care services delivered by physicians in clinics, and not on hospital services. This was reflected in the development and subsequent revisions of the fee schedule. The policy decisions which have helped to retain primary care services might provide lessons for achieving universal health coverage in low- and middle-income countries (LMICs).

Keywords: Primary Care, Medical Schools, Licensing, Specialists, Fee Schedule

Copyright: $\odot 2016$ by Kerman University of Medical Sciences

Citation: Ikegami N. Achieving universal health coverage by focusing on primary care in Japan: lessons for lowand middle-income countries. Int J Health Policy Manag. 2016;5(5):291-293. doi:10.15171/ijhpm.2016.22

\section{Article History:}

Received: 5 January 2016 Accepted: 22 February 2016 ePublished: 25 February 2016
$\mathrm{T}$ The World Health Organization's (WHO's) Consultative Group on Equity and Universal Health Coverage (UHC) stated that priority setting is unavoidable on the path to UHC..$^{1-3}$ Hard decisions must be made to deny services which are effective, but not cost-effective, in order to stay within the budget. In practical terms, it would mean prioritizing primary care in rural areas over specialist services in big urban hospitals. ${ }^{4}$ However, this would be difficult to implement given the power of the elite and of the hospital specialists. ${ }^{5}$ Moreover, even if it could be realized, the public sector has generally not been effective in delivering primary care services, so that the poor in many countries may continue to visit unlicensed practitioners. ${ }^{6,7}$ These fundamental issues have not been addressed by the "evidence-based" approach for achieving UHC. ${ }^{8}$ For these reasons, the historical decisions made in Japan that forced physicians to focus on primary care services might provide some lessons for low- and middleincome countries (LMICs). ${ }^{9}$

\section{Historical Decisions Made in Japan}

Private practitioners of traditional medicine were already well-established by the middle of the 18th century. They had the freedom to open their own practice and this principle remained essentially intact even after the government embarked on rapid modernization throughout society from 1868, mainly because the transition to Western medicine was made gradually. In 1883 , the government decided to give formal medical licenses to practitioners of traditional medicine (and their sons if 25 years or older). This assured the livelihood of existing practitioners, maintained access to service for the general population, and left intact the basic structure of the delivery system. However, from the following year, licenses could only be obtained by either graduating from designated exam-waived medical schools, or by passing the national licensure exam after studying in non-examwaived schools. The latter path was eventually closed in 1914, but medical schools below the university level continued to exist until 1952.

Although legally all those licensed had equal status, there was hierarchy among physicians based on the status of the medical school from which they had graduated. Positions in big urban hospitals were generally reserved for physicians who had graduated from higher tier schools while those from the lower tier went into private practice. Once in private practice, they could not use hospital facilities, so that despite their training as specialists, they had to focus on primary care services. This meant that the supply of specialist services was effectively constrained by the number of positions in the big hospitals. These positions remained limited because of the way hospitals developed in Japan.

Hospitals were established as a workshop for physicians to treat patients by Western medicine, and not as charity organizations for the poor. Hospitals openly listed their fees at a time when most physicians were still hesitant to do so. Most were established by physicians as extensions of their clinics. Even among the few hospitals owned by philanthropic organizations, almost all of their revenue came from patient charges. As a result, the poor generally could not access hospitals. The number of public hospitals decreased after the national government issued a decree in 1887 as part of a fiscal austerity policy which prohibited local governments from subsidizing their medical schools, and consequently their attached hospitals, which led to the closure of all but three. ${ }^{10}$ The national government restricted the function of hospitals to teaching medical students, to isolating patients with communicable diseases (initially cholera then tuberculosis), and to treating patients with combat-related injuries and diseases for the army and navy. 
The scarcity of well-equipped hospitals and universityeducated physicians led to hospital positions being controlled by the professors who headed clinical departments in medical schools. By doing so, hospitals could secure well-qualified physicians, while university professors could secure positions in well-equipped hospitals for the young doctors they have trained. This led to a hierarchy among physicians based on the status of the medical school and on the year of graduation, which, together with rivalry among universities and a focus on research, retarded the development of specialist societies based on relationship among peers.

Encouraging Primary Care and Containing Costs by the Fee Schedule

The historical development of the delivery system has been reflected and reinforced by the fee schedule for social health insurance (SHI) that was implemented in 1927. At that time, $70 \%$ of the physicians were based in clinics, and only $30 \%$ in hospitals. Moreover, since only blue-collar workers were initially covered by SHI, hospitals did not play an active role as they were focused on patients with high-income. Consequently, the original fee schedule was weighted to primary care services, and the Japan Medical Association (JMA), which was dominated by clinic-based physicians, negotiated on the behalf of all providers in its subsequent revisions.

This fee schedule was eventually adopted by the other SHI plans and was made applicable to all plans in 1959. Although the proportion of physicians-based in hospitals has increased to compose the current two-thirds, the JMA has continued to be the main actor in the revisions of the fee schedule. As a result, clinic-based physicians focusing on primary care tend to have higher income than hospital-based specialists because the specialists' organizations have remained comparatively weak. This has led to a balance of monetary and professional rewards. Although very few young physicians choose to focus on primary care, many make mid-career switches to practice primary care in clinics, tempted to do so because of the higher income and lower work load. The fact that they do not have formal training in primary care may have adversely affected quality, but health indices are generally excellent in Japan. ${ }^{11}$ The focus on primary care services and the built-in mechanism to contain costs has facilitated achieving and maintaining UHC. Costs have been contained by imposing a global budget, making item-by-item revisions, setting conditions of billing and monitoring adherence. Population coverage was achieved in 1961. The amount of out-of-pocket coinsurance was capped in 1973. Extra-billing was explicitly limited to extra charge beds, and new technology being tested for efficacy and safety in 1984. Once confirmed, the procedure will be listed. Thus, UHC has been achieved in all three dimensions. ${ }^{12}$

Possible Lessons for Low- and Middle-Income Countries Compared with the situation in Japan long ago, the benefits of medical care have increased and the public is more aware of the technology available in big hospitals. Moreover, unlike Japan, many LMICs have a colonial legacy of public hospitals that were established to provide specialist services ostensibly to all. It is no wonder that these hospitals are over-crowded and under-staffed, thus providing a valid reason for allocating more resources. However, policy-makers must recognize that resources must be prioritized on primary care so that all are assured access to basic services. The following are suggestions for LMICs to facilitate this process based on Japan's experience:

1. Integrate unlicensed practitioners into the healthcare system in exchange for adhering to basic regulations on price, quality and information disclosure in order to increase their accountability. Market-based approaches are likely to be more effective than interventions that rely on training. ${ }^{13}$ Where assistant doctors exist, they should be given opportunities to obtain full licenses. By doing so, the relative share of those providing primary care would increase and their power would be enhanced.

2. Reduce the public funding of big public hospitals. A fiscal crisis could be the window of opportunity for doing so. At the same time, restrict specialists-based in big hospitals from practicing in private clinics, and private-practice physicians from using the equipment and resources of public hospitals. As opportunities to deliver specialist services decreases, more physicians would be forced to focus on primary care.

3. Design a fee schedule that lists primary care services, ear-mark their funding and pay public and private providers on the same basis. Encourage the development of a primary care providers' association and make it the key actor in the revisions of the fee schedule. Gradually expand the services and drugs listed to cover specialist services within the fiscal space available. This expansion should be in line with the institutional capacity to regulate fees and monitor adherence to the conditions set. ${ }^{14}$

The key to reform is managing the expectations of the public on the services they are entitled to, and of the physicians on their income and practice conditions. Given the resource constraints, only a restricted range of specialist services can be made accessible to most of the population. The government should deflate the expectations of the public and the physicians and focus on expanding primary care services.

Ethical issues

Not applicable.

Competing interests

Author declares that he has no competing interests.

Author's contribution

$\mathrm{NI}$ is the single author of the paper.

\section{References}

1. Evans DB, Etienne C. Health systems financing: the path to universal coverage. Bull World Health Organ. 2010;88:402. doi:10.2471/BLT.10.078741

2. World Health Organization (WHO). Making fair choices on the path to universal health coverage: Final report of the WHO Consultative Group on Equity and Universal Health Coverage. Geneva: WHO; 2014.

3. Norheim OF. Ethical perspective: five unacceptable trade-offs on the path to universal health coverage. Int J Heath Policy Manag. 2015;4(11):711-714. doi:10.15171/ijhpm.2015.184

4. Starfield B, Shi L, Macinko. Contribution of primary care to health systems and health. Milbank Q. 2005,83(3):457-502. doi:10.1111/j.1468-0009.2005.00409.x 
5. Ikegami N, Campbell JC. Dealing with the medical axis-ofpower: the case of Japan. Health Economics, Policy and Law. 2008;3:107-113. doi:10.1017/S1744133108004428

6. Filmer D, Hammer JS, Pritchett LH. Weak links in the chain: a diagnosis of health policy in poor countries. World Bank Research Observer. 2000;15(2):199-224. doi:10.1093/wbro/15.2.199

7. George A, lyer A. Socially embedded informal health providers in northern Karnataka, India. Soc Sci Med. 2013;96:297-304. doi:10.1016/j.socscimed.2013.01.022

8. Lewin S, Lavis JN, Oxman AD, et al. Alma-Alta: Rebirth and Revision 2: Supporting the delivery of cost-effective interventions in primary health-care systems in low-income and middleincome countries: an overview of systemic reviews. Lancet. 2008;372(9642):928-939. doi:10.1016/s0140-6736(08)61403-8

9. IkegamiN.UniversalHealthCoveragefor InclusiveandSustainable Development - Lessons from Japan. http://documents. worldbank.org/curated/en/2014/09/20278271/universal-healthcoverage-inclusive-sustainable-development-lessons-japan.
Published 2014.

10. Sakai S. History of Medical Care in Japan. Tokyo: Tokyo Shoseki; 1982:432

11. Hashimoto $\mathrm{H}$, Ikegami $\mathrm{N}$, Shibuya $\mathrm{K}$, et al. Universal health care at 50 years 3 . Cost containment and quality of care in Japan: is there a trade-off? Lancet. 2011;378(9797):1174-1182. doi:10.1016/S0140-6736(11)60987-2

12. Ikegami N, Yoo BK, Hashimoto $\mathrm{H}$, et al. Japanese universal health coverage: evolution, achievements, and challenges. Lancet. 2011;378(9796):1106-1115. doi:10.1016/S01406736(11)60828-3

13. Shah NM, Brieger WR, Peters DH. Can interventions improve health services from informal private providers in low and middleincome countries? A comprehensive review of the literature. Health Policy Plan. 2011;26:275-287.

14. Ikegami N. Fee-for-service payment - an evil practice that must be stamped out? Int J Heath Policy Manag. 2015;4(2):57-59. doi:10.15171/ijhpm.2015.26 